\title{
Spatially Resolved Femtosecond Pump-Probe Spectroscopy in Broad-Area Semiconductor Lasers
}

\author{
Joachim Kaiser, Ingo Fischer, Wolfgang Elsäßer, Senior Member, IEEE, Edeltraud Gehrig, and Ortwin Hess
}

\begin{abstract}
We investigate the ultrafast gain dynamics in broad-area semiconductor lasers with particular emphasis on spatial and spatiotemporal effects. We present a spatially resolved femtosecond pump-probe experiment which allows us to measure the compression and recovery of the gain with 250 -fs temporal and $15 \mu \mathrm{m}$ spatial resolution. We find a significant spatial variation of the gain recovery time across the lateral laser coordinate indicating an influence of the extended laser structure on the ultrafast carrier relaxation. Moreover, we are able to follow the spatiotemporal relaxation of the ultrafast spatiospectral gain saturation within the extended semiconductor active area. We find diffusion-like broadening of the locally suppressed gain on two distinct ultrafast timescales, within several picoseconds and several tens of picoseconds, resulting from an interplay between intraband relaxation, spatial holeburning, and light propagation. Supported by microscopic modeling, our results provide insight into the different mechanisms and timescales associated with the spatiotemporal carrier dynamics. These findings are essential for the design of laterally extended semiconductor active devices for ultrafast optical signal processing applications.
\end{abstract}

Index Terms-Broad-area lasers (BALs), carrier dynamics, femtosecond spectroscopy, gain saturation, pulse propagation, semiconductor lasers, ultrafast optics.

\section{INTRODUCTION}

$\mathbf{I}$ $\mathrm{N}$ SEMICONDUCTOR lasers and amplifiers, the ultrafast optical properties are strongly determined by intraband carrier dynamics. This takes place on timescales ranging from several picoseconds to femtoseconds and is responsible for significant gain and index nonlinearities [1], [2]. Investigating these fast or even ultrafast timescales is thus essential for getting a microscopic understanding of the relevant mechanisms that determine the interaction between the carrier system and the optical field. In addition, these processes are regarded of high practical relevance for future applications in optical communication and optical signal processing with semiconductor optoelectronic devices. On the one hand, they can generate complicated temporal and spectral distortions in propagating ultrafast optical pulses [3]-[8]. On the other hand, however, they can also enable the realization of devices for subpicosecond all-optical signal pro-

Manuscript received November 4, 2005; revised January 19, 2006.

J. Kaiser and W. Elsäßer are with the Institute of Applied Physics, Darmstadt University of Technology, 64289 Darmstadt, Germany (e-mail: joachim.kaiser@physik.tu-darmstadt.de; elsaesser@physik.tu-darmstadt.de).

I. Fischer was with the Institute of Applied Physics, Darmstadt University of Technology, 64289 Darmstadt, Germany. He is now with Department of Applied Physics and Photonics, Vrije Universiteit Brussels, 1050 Brussels, Belgium (e-mail: ingo.fischer@physik.tu-darmstadt.de).

E. Gehrig and O. Hess are with the Advanced Technology Institute, School of Electronics and Physical Sciences, University of Surrey, Guildford, Surrey GU2 7XH, U.K. (e-mail: e.gehrig@ surrey.ac.uk; o.hess@ surrey.ac.uk).

Digital Object Identifier 10.1109/JQE.2006.871556 cessing such as wavelength converters, switches, or demultiplexers which are based on ultrafast cross gain modulation or four-wave mixing [9].

In the past, the carrier dynamics has been extensively investigated by femtosecond pump-probe methods in semiconductor amplifiers. Spectral holeburning and carrier heating have been identified as main contributions to the ultrafast nonlinear gain. The associated relaxation of the nonequilibrium carrier distributions via carrier-carrier and carrier-phonon scattering has been measured; the obtained time constants are typically in the range of 100-300 fs and 0.4-1 ps for thermalization and temperature relaxation of the carrier systems within the bands, respectively [10]-[16].

Here, we expand the investigations of the ultrafast gain dynamics on spatially extended devices, investigating broad-area semiconductor lasers (BALs). These are specific edge-emitting semiconductor lasers, in which the lateral extension is widened up to typically $100 \mu \mathrm{m}$ to obtain high power output. Previous investigations of the emission dynamics of these lasers have shown, that the spatial extension leads to a complex spatiotemporal dynamics on pico- and nanosecond timescales caused by local effects and nonlinear interaction between the carriers and the light field [17]. These effects deteriorate the output significantly, but the combination of the spatial extension with the nonlinear interaction can also be used for novel photonic devices. Utilization of BALs as, e.g., phase conjugate mirrors by spatially extended, nondegenerate four-wave mixing in the gigahertz range has already been demonstrated [18], [19]. In this paper, we experimentally investigate whether the specific laser structure of BALs also has an influence on processes on ultrafast timescales-indications that laser action can influence the intraband relaxation rates have already been found in a comparison between lasers and amplifiers [20]—and whether, in particular, spatial dependencies and spatiotemporal dynamics can be found in the pico- and subpicosecond regime.

Spatially resolved microscopic modeling of broad-area semiconductor devices has shown that combined spatiospectral nonlinearities exist and that they play an important role in ultrafast pulse propagation [21]. They lead to complex spatiotemporal pulse-shaping and can be used for soliton generation and ultrafast phase conjugation [22], [23]. Knowledge of spatial dependencies and spatiotemporal effects on ultrafast timescales obtained from experimental measurements is thus necessary to allow comparison with the results predicted by theoretical modeling. Moreover, it is also essential for the design of future optoelectronic devices for optical signal processing that make use of the ultrafast spatiospectral nonlinearities in spatially extended semiconductor active areas. 
The paper is organized as follows. In Section II, we present a spatially resolved femtosecond pump-probe setup, which allows investigations of the gain dynamics in BALs with 250 -fs temporal and $15-\mu \mathrm{m}$ spatial resolution. In Section III, we present the experimental results, which have been obtained using two different $100-\mu \mathrm{m}$ BALs. We first examine the ultrafast response of the gain in a conventional collinear pump-probe measurement and identify the relevant contributions to gain saturation and their relaxation rates. Second, we measure the lateral dependence of the picosecond temperature relaxation of the carrier system, which indeed exhibits a significant variation across the laser lateral extension. Third, we use spatially separated pump and probe pulses to map the ultrafast spatiotemporal relaxation of a locally induced spatiospectral gain saturation and the relaxation of the subsequent spatial hole. These experimental investigations are complemented by microscopic modeling of the spatially resolved gain following a femtosecond pulse excitation, which substantiates the experimentally observed interplay between intraband relaxation, spatial holeburning and light propagation occurring on different timescales in the picosecond regime and all together being relevant for the spatiotemporal carrier dynamics.

\section{DeVices AND EXPerimental SetuP}

The measurements are performed on two commercial BALs from Coherent and Spectra Diode Labs (called types "A" and "B"). They have an InGaAsP and an AlGaAs quantum-well active area with a gain maximum around $800 \mathrm{~nm}$, laser A emitting TM, and laser B TE-polarized light. The stripe width is $100 \mu \mathrm{m}$ with index guiding in both laser types, the lengths of the lasers are 1 and $0.75 \mathrm{~mm}$, with threshold currents of 365 and $380 \mathrm{~mA}$, respectively.

The experimental setup is based on a conventional two-pulse, cross-polarized pump-probe scheme, which is depicted in Fig. 1. Optical pulses from a Ti:sapphire laser (pulsewidth $150 \mathrm{fs}$, repetition rate $75 \mathrm{MHz}$, wavelength tunable) are separated into pairs of pump and probe pulses at the first beamsplitter. At the second beamsplitter pump and probe beam are recombined. They are injected through the low-reflectivity (about 5\%) front facet and propagate through the laser, which is operated close to threshold. The laser is mounted on a heat sink that is actively stabilized to room temperature. The half-wave plate in the pump beam rotates the polarization direction of the pump by $90^{\circ}$ with respect to the probe beam so that, after having propagated through the laser, it can be blocked from the probe pulse by the polarizer. The polarization of the pump pulse is chosen to correspond to the polarization of the laser emission. The intensity of the transmitted probe signal, which is in addition modulated at $1 \mathrm{kHz}$ by the optical chopper in the probe beam, is detected by a p-i-n photodiode, whose signal is measured by a lock-in amplifier. A translation of the retroreflector in the pump beam allows to vary the delay $\tau$ between pump and probe pulses. Recording the intensity of the transmitted probe signal as function of the delay $\tau$ is a direct measure of the transient gain response after the excitation of the carrier system with the pump pulse. The pulse energies of the pump and the probe pulses are 100 and $2 \mathrm{pJ}$, respectively. The

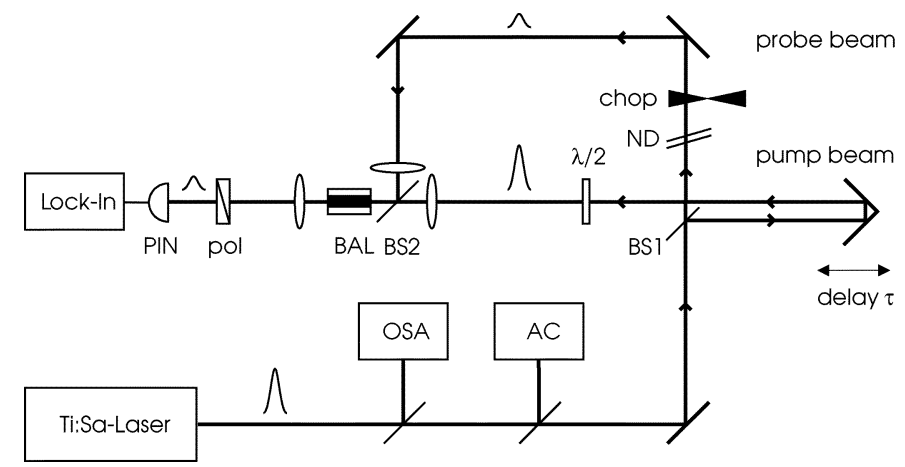

Fig. 1. Schematic of the experimental pump-probe setup. OSA=optical spectrum analyzer, $\mathrm{AC}=$ autocorrelator, $\mathrm{BS} 1 / 2=$ =beamsplitter, $\mathrm{ND}=$ neutral density attenuator, $\lambda / 2=$ halfwaveplate, pol=polarizer, PIN=photodiode.

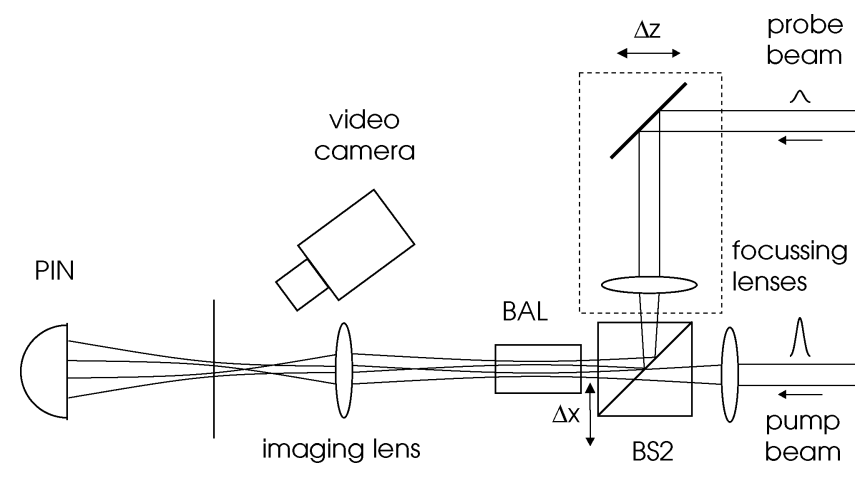

Fig. 2. Detailed view of the spatially resolved measurement.

overall temporal resolution of the setup, considering dispersive broadening and pulse walkoff due to birefringence during propagation in the laser, can be estimated to $250 \mathrm{fs}$.

The realization of the spatial resolution is depicted in Fig. 2, which shows the pulse injection and beam propagation within the laser in more detail. In order to obtain parallel propagating beams, each having a focus inside the BAL, the combination of the beams by the beamsplitter cube is done after focussing of each beam. The beam waists of the pump and the probe beam are placed inside the laser at the same $z$-position ( $z=$ propagation direction). The lateral positioning of the pump beam can be performed by a lateral movement of the laser, the lateral positioning of the probe beam with respect to the pump beam is achieved by a combined translation $\Delta z$ of the mirror and the focussing lens in the probe beam, which transforms to a parallel displacement of the probe beam in $x$-direction by $\Delta x=\Delta z$. After propagation through the laser, both beams are imaged by an imaging lens onto a screen observed by a videocamera, which allows to adjust and control the spatial positioning of the beam waists inside the laser. For detection, the screen is removed and the probe beam falls onto the $p-i-n$ photodiode while the pump beam is blocked as described above.

The spatial resolution is determined by the beam diameters inside the laser and requires a compromise between a small beam waist and a large Rayleigh length. In our case, using lenses with 11-mm focal length the beam waist amounts to 10 $\mu \mathrm{m}$ with a Rayleigh length of $250 \mu \mathrm{m}$. Considering the whole passage through the laser, the spatial resolution can, therefore, 

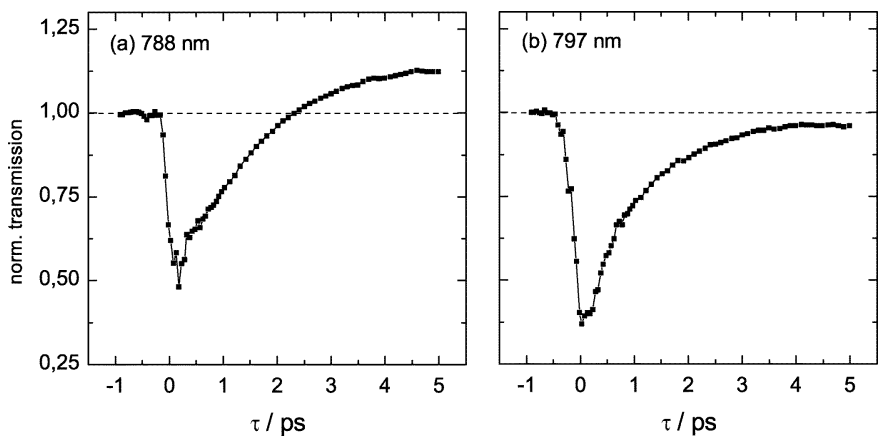

Fig. 3. Normalized probe transmission as function of pump-probe delay $\tau$ for 788 and $797 \mathrm{~nm}$ center wavelength of the pump and probe pulses.

be estimated to roughly $15 \mu \mathrm{m}$, which should, however, be modified due to amplification/absorption and self-focussing effects during propagation in the laser. To experimentally measure the spatial resolution, we use zero delay pump and probe pulses. Under zero delay, there is a strong reduction of the transmitted probe intensity due to two-photon-absorption from the probe and the pump pulse [1], [13]. Measuring this two-photon-absorption contribution as function of the lateral separation $\Delta x$ of the beams, therefore, maps the spatial overlap of the two beams. Doing so, we obtain a Gaussian shaped probe signal reduction as function of $\Delta x$ with a full-width at half-maximum (FWHM) of $24 \mu \mathrm{m}$, corresponding to a convolution of two beams with an effective FWHM diameter of $17 \mu \mathrm{m}$.

\section{RESUlTS AND DisCUSSION}

\section{A. Ultrafast Gain Compression and Relaxation}

In this section, we first analyze and discuss the ultrafast transient evolution of the gain following the excitation with the pump pulse without special attention on spatial effects. Both pulses coincide spatially and are injected centrally into the laser. Fig. 3 shows the obtained transmission of the probe pulse as function of the pump-probe delay $\tau$ in laser A during the first few picoseconds after the excitation. The transmission is normalized to the value for $\tau<0$, i.e., transmission without additional excitation. The BAL is biased to $95 \%$ of its threshold value. The center wavelengths of the pump and the probe pulses are $788 \mathrm{~nm}$ [Fig. 3(a)] and $797 \mathrm{~nm}$ [Fig. 3(b)], thus corresponding to absorption and amplification within the semiconductor gain spectrum, respectively.

Both curves show the typical qualitative time evolution of the gain, which is commonly found in semiconductors and which we shall briefly discuss. At $\tau=0$ there is a strong instantaneous reduction of transmission which is always observed in pump-probe measurements and is mainly due to two-photonabsorption, when one photon from the pump and one photon from the probe pulse are absorbed simultaneously. It thus requires temporal overlap of both pulses and has been used to determine the zero-delay position. Following the evolution, the transmission signal remains below its undisturbed value, indicating ultrafast gain compression, which relaxes to a constant value after several picoseconds. This value results from a net change of the carrier density and is thus positive compared to $\tau<0$ in the absorption regime [Fig. 3(a)], where the pump pulse generates additional carriers. Correspondingly, in the gain regime the net carrier change is negative [Fig. 3(b)]. This change in the net carrier density relaxes on a much slower nanosecond timescale, which is not investigated here. Yet it happens faster than the repetition period of the injected pulses of about $13 \mathrm{~ns}$ so that no carrier accumulation occurs over successive pulses. Therefore, for negative delay $\tau$, we indeed observe the transmission through the undisturbed gain.

The most interesting part of the transmission curves is the gain compression and relaxation during the first few picoseconds as it contains the contributions of nonlinearities resulting from changes in the spectral gain distributions such as spectral holeburning and carrier heating and their intraband relaxation. For a quantitative analysis of the relaxation a fit with a sum of two exponential decays has been used, which in contrast to a single exponential decay is able to fit the data very accurately and has been used to fit experimental transmission curves before [1]. By several tests, we carefully chose the starting times of the fit to avoid an influence of the two-photon-absorption around zero delay. The analysis has been performed for a range of pump-probe wavelengths from 785 to $800 \mathrm{~nm}$. As a result, we find two relaxation times amounting to $T_{\text {slow }}=1.2 \pm 0.2 \mathrm{ps}$ and $T_{\text {fast }}=300 \pm 200 \mathrm{fs}$. They are independent of the pump-probe wavelength, which indicates that the fit works properly and we do not have a systematic error, e.g., due to the two-photon-absorption contribution. The amplitude of the slow exponential decay term is negative for each pump-probe wavelength, whereas that of the fast exponential decay term changes sign from positive to negative with increasing wavelength. We can conclude that the negative contribution to the gain which relaxes with a time constant of 1.2 ps results from carrier heating. Heating occurs in the absorption as well as in the gain regime by absorption of high energy carriers or by selective removal of low energy carriers, respectively. In contrast, the fast subpicosecond relaxation is an indication of spectral holeburning and its thermalization, which increases the gain at its spectral position in the absorption regime and reduces it in the gain regime.

A corresponding analysis of the experimental data in laser B yields a carrier heating relaxation time of $T_{\text {slow }}=1.4 \pm 0.2 \mathrm{ps}$. A fast component indicating spectral holeburning could not be extracted with sufficient certainty for this laser. A comparison of the obtained values for $T_{\text {slow }}$ of 1.2 and $1.4 \mathrm{ps}$ with previous examinations of semiconductor amplifiers shows that the gain relaxation is slightly slower than what was typically found (being in the range of $0.6-1 \mathrm{ps)} \mathrm{[1],} \mathrm{[10],} \mathrm{[13],} \mathrm{[15].} \mathrm{This}$ seems to be in agreement with [20], where an increase of the gain relaxation time from 0.7 to 0.9 ps has been found when converting a special quantum well device from an amplifier to a laser. As a reason for the slowing down of the gain relaxation, it has been suggested that additional heating of the carrier system occurs due to carrier capture from the barrier regions as a consequence of the enhanced stimulated emission. However, as we are dealing with BALs here, other contributions may occur. For a further analysis and interpretation of the relevance of the spatial extension we investigate the gain relaxation within the BALs with spatial resolution in the following. 


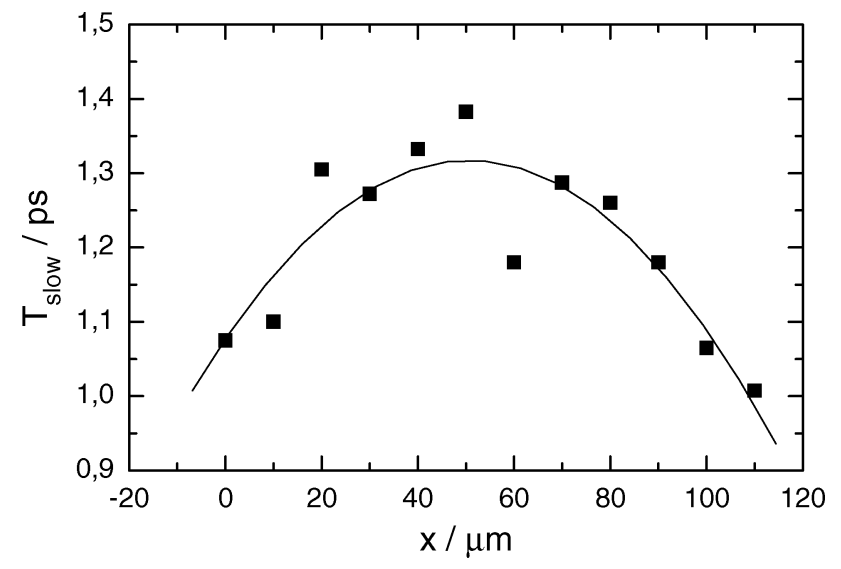

Fig. 4. Carrier heating relaxation time $T_{\text {slow }}$ as function of the lateral position $x$ in laser A.

\section{B. Spatial Dependence of the Intraband Relaxation}

To measure the lateral dependence of the ultrafast gain relaxation, pump and probe pulses are sent through the laser collinearly as in conventional pump-probe measurements. However, the lateral extension of each of the beams is confined to a small width within the laser during propagation. This common position of the pump and the probe beam can be varied in lateral direction as described in Section II. As a result, we obtain a set of transmission curves, like those shown in the previous subsection, for different lateral positions within the laser stripe. From these curves, we again determine the gain relaxation times. In the following, we concentrate on the slower relaxation time $T_{\text {slow }}$, as the uncertainties of $T_{\text {fast }}$ are too large to allow significant statements concerning their spatial dependence.

Fig. 4 depicts the slow gain relaxation time $T_{\text {slow }}$ evaluated from the transmission curves as a function of the lateral coordinate $x$ across the $100-\mu \mathrm{m}$ broad laser stripe of laser A at $\lambda=796$. For each position, three independent measurements have been performed and averaged to improve the significance of the data. We find that there is indeed a measurable spatial variation of the gain relaxation time. The relaxation is slowest at the center of the laser and drops from 1.3 to 1.0 ps approaching the left and right side of the active stripe. The continuous line is a guide to the eye obtained from a quadratic fit of the data, which has been arbitrarily chosen.

These results seem to affirm the effect of an increase of the gain relaxation under stimulated emission. Under operation closely below threshold, the BAL exhibits a spatial intensity profile of the light which has a maximum at the center of the stripe and decreases to both sides. The measured gain recovery time apparently corresponds to the spatial distribution of the light intensity.

We note that other parameters vary across the active area as well. In particular, we expect a laterally varying temperature distribution across the laser stripe. This temperature distribution most likely affects the picosecond gain relaxation that is associated with carrier temperature relaxation due to phonon scattering. In Fig. 5, it is clearly shown that the spatial variation of the relaxation time $T_{\text {slow }}$ does not necessarily behave as displayed in Fig. 4 . Fig. 5 depicts the relaxation time $T_{\text {slow }}$

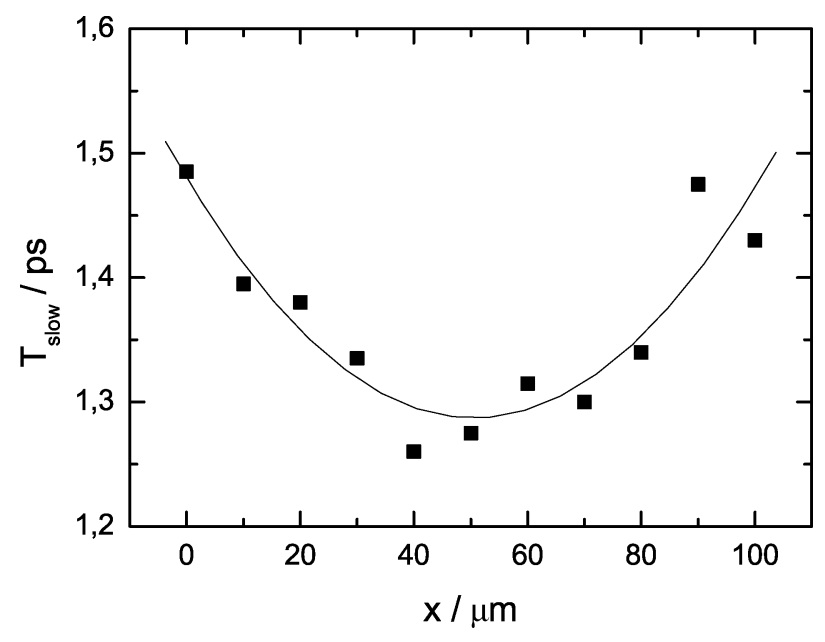

Fig. 5. Carrier heating relaxation time $T_{\text {slow }}$ as function of the lateral position $x$ in laser B.

as a function of the lateral coordinate $x$, however, for laser $\mathrm{B}$, which is driven closely below threshold. Here, each data point is based on two independent measurements taken for $\lambda=794$ and $797 \mathrm{~nm}$. We find a distribution that exhibits the contrary dependence compared to that in laser A. The relaxation time is fastest at the center of the laser and increases by 0.2 ps approaching the boundaries at both sides.

We would like to note, that as we use several independent measurements for the data presented in Figs. 4 and 5, the actual error bars are less than $\pm 0.2 \mathrm{ps}$. We find that deviations from the fitted curves are remarkably low and in particular much less than $0.2 \mathrm{ps}$. So from the experimental data we can conclude that there is indeed a spatial dependence of the carrier relaxation within the active stripe. Moreover, considering the different results obtained in the two lasers, obviously different effects with opposite sign influence the carrier temperature relaxation. Depending on the relative strength the resulting combined effect can, therefore, have opposite sign, too. At this point, we cannot clarify, which effects actually influence the picosecond gain relaxation to which amount. We suggest that stimulated emission as well as the lattice temperature can play an important role. For the latter mechanism, in particular, we have to consider the different types of strain in the quantum-well layers in lasers A and B, as becomes apparent in the different polarization of the emitted light. Laser A is a tensile-strained InGaAsP device compared to the AlGaAs material of laser B. So, an increase of the local temperature could have an opposite effect on the total amount of strain, which might explain the observed contrary behavior of the relaxation time in both lasers. To complement the presented experiments, we have also performed measurements of the dependence of the carrier relaxation time on pump current. Here, we also find that at least two opposite contributions seem to be involved. We find over the whole range of pump current a tendency of a decrease of the carrier relaxation time. In addition at pump currents close to but below threshold we find a (then dominating) increase of the relaxation time. This could be interpreted as an indication that heating of the device tends to decrease the relaxation time and another effect (amount of stimulated emission or carrier density, which increases most with pump current closely below threshold) has an increasing effect. 


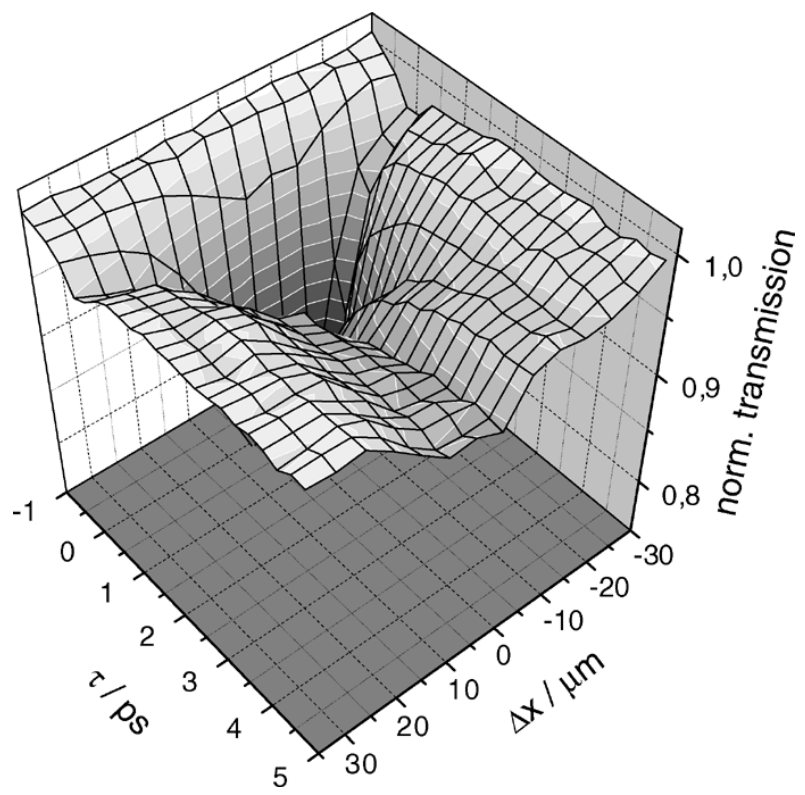

Fig. 6. Normalized probe transmission as function of delay $\tau$ and lateral pump-probe separation $\Delta x$ in laser A.

Yet, we cannot definitely separate the different effects, so further experimental and theoretical investigations will be necessary, in particular of the carrier-phonon interaction in these devices, which is mainly responsible for $T_{\text {slow }}$ relaxation. Nevertheless, the results show that the ultrafast gain recovery due to intraband carrier relaxation is actually not alone a material property but is significantly influenced by the device operation. The results reveal that a spatial dependence of the picosecond gain relaxation indeed occurs. This will affect ultrafast spatiotemporal pulse propagation or four-wave mixing in spatially extended semiconductor active areas and has to be taken into account in modeling and design of devices for ultrafast optical signal processing. As mechanisms of opposite effect are involved, experimental measurements of the relaxation behavior are indispensable and can serve as input for modeling of the spatially extended devices.

\section{Spatiotemporal Gain Dynamics}

As a last step of the investigations of the ultrafast nonlinear gain, we spatially separate the pump and the probe beam. By variation of the lateral position of the probe beam we can measure the spatiotemporal relaxation of the excitation by the pump pulse that is done at a fixed position. For the pump pulse we have chosen the center of the active area as reference position. Fig. 6 shows, in a three-dimensional (3-D) plot, the normalized gain as function of pump-probe delay $\tau$ and lateral distance between pump and probe beam $\Delta x$ at $\lambda=798 \mathrm{~nm}$, thus being in the gain regime. The zero delay has been defined from the central curve $(\Delta x=0)$. For the other curves the same zero delay point has been used corrected by $\Delta x / c$ due to the change of the length in the probe beam path (see Fig. 2). We find an ultrafast local compression and relaxation of the gain following the excitation, which for $\Delta x=0$ exhibits the features discussed in Section III-A indicating the occurrence of spectral holeburning and carrier heating. In addition, in this plot we clearly see the

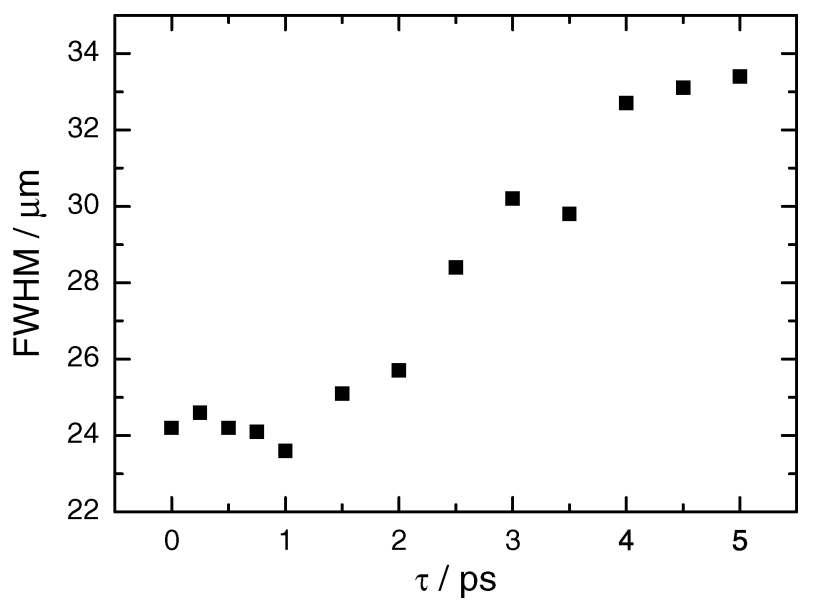

Fig. 7. Evolution of the spatial width of the probe signal reduction as function of delay $\tau$ obtained from the experimental data shown in Fig. 6.

local character of the spectral nonlinearities. We thus directly map simultaneous spatiospectral holeburning and spatiospectral carrier heating. Due to the different timescales for spectral (intraband) and spatial relaxation, the spatiospectrally reduced gain relaxes into a purely spatial hole. The spatial profile after 5 ps shows the spectral hole created by carrier depletion due to the injected pulse. In contrast to the spectral relaxation, the subsequent relaxation of the spatial hole by carrier diffusion and intraband relaxation is expected to happen on much slower timescales, which will be discussed below.

Fig. 7 depicts the width of the dip in the normalized spatial gain depending on $\tau$. By this analysis of the spatial profile we find that the width of the dip at $\tau=0$ is $24 \mu \mathrm{m}$. Interestingly, the width increases continuously during the ultrafast relaxation within the first few picoseconds and amounts to $33 \mu \mathrm{m}$ for the spatial hole after 5 ps. Thus, we actually find, in addition to the temporal relaxation, a spatial relaxation of the gain compression on the timescale of a few picoseconds, which of course cannot be explained by the much slower carrier diffusion occurring in the nanosecond regime. Obviously, the intraband spectral relaxation of the created spatiospectral hole and the subsequent spatiospectral carrier heating occurs on the fastest timescale at the center of the excitation, where the deviation from the quasi-equilibrium distribution is highest, and increasingly slower toward the sides. Another influence on the observed ultrafast spatial broadening should result from the two-photon contribution in combination with the saturated excitation by the pump pulse. For $\tau=0$, the spatial profile of the dip is, as mentioned before, a convolution of the pump and the probe spatial profiles. In contrast, the width of the measured spatial hole after $\tau=5 \mathrm{ps}$ is a convolution of the probe beam profile with the actual spatial hole which is, however, broadened compared to the pump beam profile because of the saturated gain that flattens the Gaussian profile. During the ultrafast spectral relaxation, this spatial widening due to the nonlinear excitation becomes more and more important, which in effect leads to an ultrafast broadening of the locally reduced gain. In summary, the ultrafast nonlinear gain exhibits a spatiotemporal relaxation that effectively resembles an ultrafast diffusion-like broadening mechanism, but actually results from the interplay between the 


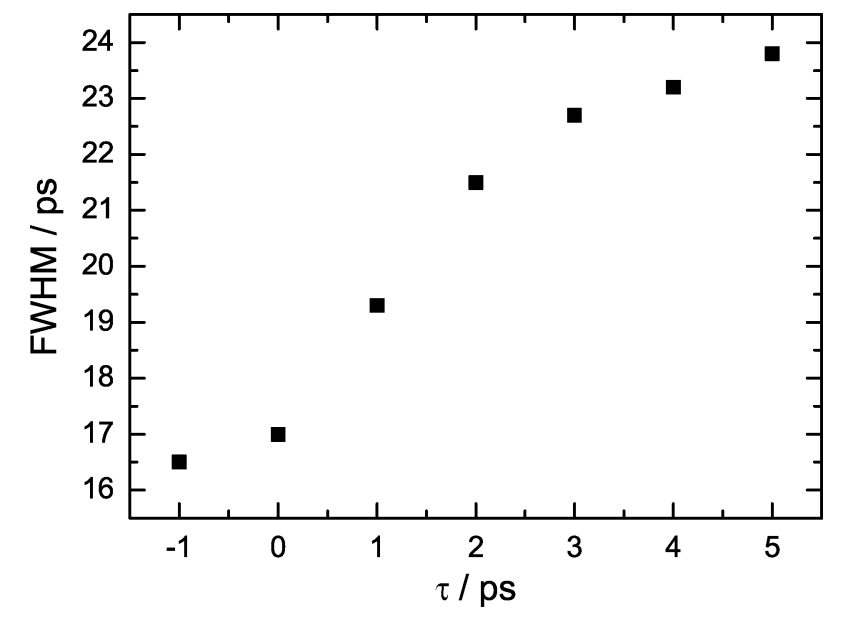

Fig. 8. Evolution of the spatial width of the gain reduction as function of delay $\tau$ obtained from theoretical modeling.

two-photon contribution at zero-delay, the saturated gain, and the intraband relaxation of the spatiospectral hole into the spatial hole.

We compare the experimental results with results of microscopic modeling. The theoretical description is based on spatially resolved Maxwell-Bloch equations that describe the spatiotemporal light field and carrier dynamics in spatially extended semiconductor lasers [21]. The theory includes in particular counterpropagating light waves, light diffraction, the full polarization dynamics (including dynamic gain saturation and induced index dynamics), dynamic self-focusing, carrier diffusion, and scattering. The equations of motion are integrated in the lateral and longitudinal direction thereby taking into account the spatially and temporally varying light-matter coupling. The vertical direction enters the equation via effective parameters. These are, in particular, the optical confinement factor and the material gain resulting from the vertical epitaxial structure of the device. The latter enters the Bloch equations as an effective material gain parameter. A thorough adjustment of the material gain to a given experimental device has to be realized by a systematic comparison of theoretical and experimental dependencies of output power on injection current density. This is necessary since the physical properties of a semiconductor laser depend on (often unknown, as in our case) details of the material and epitaxial structure or may even vary from device to device.

In the simulations, the injection of the pulse is realized via the boundary conditions of the light fields. Similar to the experiments, a 15- $\mu \mathrm{m}$-broad 150-fs-long pulse has been used, injected into a $100-\mu \mathrm{m}$ broad and $1-\mathrm{mm}$-long BAL. Differently to the experiments, no additional probe pulse was simulated, instead the spatially resolved gain at the spectral position of the excitation pulse for different times after the excitation is directly evaluated. This provides the same information as in the experiments, where the probe pulse is used to measure the instantaneous gain, however, artifacts resulting from a direct interaction between the two pulses are missing in the simulation.

Fig. 8 depicts the temporal evolution of the locally suppressed gain by a pump pulse analogous to Fig. 7 , in this case obtained from the modeling. A significant broadening of the dip created
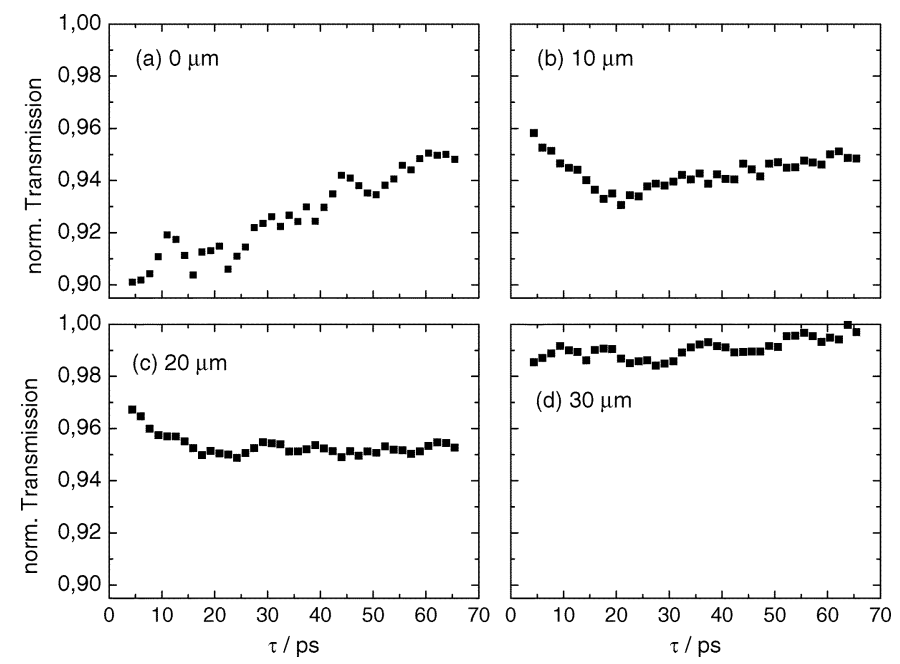

Fig. 9. Normalized probe transmission as function of delay $\tau$ for different lateral pump-probe separations $\Delta x=0,10,20,30 \mu \mathrm{m}(\mathrm{a}-\mathrm{d})$ in laser A.

by the pump pulse in the gain can be found. Within the first $5 \mathrm{ps}$ after the excitation the gain broadens from 17 to $24 \mu \mathrm{m}$. Though the exact behavior of the spatiotemporal gain relaxation depends on the injected pulse energy and the pump current, this is an increase roughly by the same amount as in the experimental measurement of Fig. 7. (Note, that the convolution with the spatially extended probe beam is not included here.) From the fact, that the zero-delay dip due to two-photon-absorption is not included in the theoretical results, we can conclude that this pump-probe specific artifact is at least not solely responsible for the observed ultrafast broadening. Even though this effect alone would be worth being considered, as in real-world experiments the direct interaction of the two coincident pulses is unavoidable.

What remains after $\tau=5$ ps can be regarded as a purely spatial hole in the carrier density. In order to investigate the temporal evolution of this spatial hole, which is expected to take place on significantly slower timescales, we expand our spatially resolved pump-probe measurements to longer time windows and record the probe transmission for a pump-probe delay of up to $\tau=70$ ps. Fig. 9 shows experimentally obtained probe transmission curves as function of the pump-probe delay starting from $\tau=5 \mathrm{ps}$, thus now neglecting the ultrafast evolution during the first few picoseconds at $\lambda=798 \mathrm{~nm}$. To investigate spatiotemporal effects, four traces have been recorded for different lateral pump-probe separations $\Delta x$ from 0 to $30 \mu \mathrm{m}$ [Fig. 9(a)-(d)]. For zero separation, we find a steady increase of the probe transmission signal corresponding to a decrease of the spatial hole at its center. At $\Delta x=10 \mu \mathrm{m}$, the signal in contrast drops until reaching a minimum value at about $\tau=20 \mathrm{ps}$ and then increases again. This means that the local carrier density first decreases and then starts to build up again. The curve at $20-\mu \mathrm{m}$ separation shows a similar behavior, but less pronounced and with the minimum appearing even later than before. At $\Delta x=30 \mu \mathrm{m}$ almost no effect is visible.

To get a better understanding of the presented behavior, we again performed numerical simulations of the spatiotemporal evolution of the gain after pulse excitation within $70 \mathrm{ps}$. The results of the simulations are depicted in Fig. 10. They show, as compared to Fig. 9, the relative change of gain in arbitrary units 

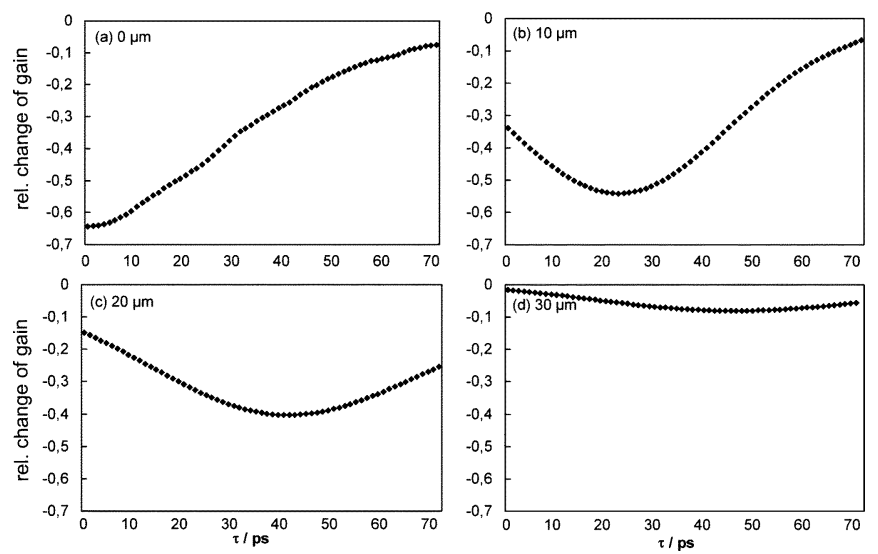

Fig. 10. Relative change of probe gain as function of delay $\tau$ for different lateral pump-probe separations $\Delta x=0,10,20,30 \mu \mathrm{m}(\mathrm{a}-\mathrm{d})$ obtained from theoretical modeling.

as function of the time $\tau$ after pulse excitation with a $15-\mu \mathrm{m}$ broad pulse at the center of the BAL. The gain has been evaluated by longitudinal averaging of the spatially dependent gain for each lateral position. Again four curves are plotted for different lateral separations between the position of excitation and detection. The calculated curves qualitatively show a strikingly similar behavior to the measured curves of Fig. 9. Because of the much better signal-to-noise ratio, the main features are much better observable than in the experiments. The results show clear evidence of a diffusion-like behavior: while at the center of the dip the signal steadily increases, it runs through a minimum for positions away from the center. The farther away the position from the center, the later the minimum arrives and the weaker it is pronounced. In conclusion, we obviously observe a diffusion-like relaxation behavior of the spatial hole created by the pump excitation which happens on a timescale of several ten picoseconds. Note, that this is an intermediate timescale slower than the ultrafast intraband relaxation, however much faster than the nanosecond interband relaxation or carrier diffusion. If we evaluate the effective diffusion constant from our experimental or modeling results, we obtain a value which is by a factor of about 1000 , which is too high compared to typical carrier diffusion constants in semiconductors. This intermediate timescale rather corresponds to the typical timescales of light propagation within the laser. So we conclude that it is in fact the presence of the light propagating internally in the laser in conjunction with the spectral and spatial carrier relaxation, that is responsible for the specific picosecond spatiotemporal carrier relaxation in the BAL. From the simulations, this conclusion is further supported by the fact that only a model that explicitly takes into account propagation effects can describe the observed behavior, whereas an application of a corresponding longitudinally averaging model, i.e., a meanfield approach of the Maxwell-Bloch equations, leads to a spatial hole burning that relaxes on much longer time scales in the nanosecond regime.

The interplay of the different timescales in these devices and the occurrence of the picosecond relaxation can be summarized as follows. Generally, the dynamics of semiconductor lasers is strongly determined by the complex interplay of spatiotemporal interactions occurring on time scales ranging from the femtosecond up to the nanosecond regime. The carrier distributions within the bands of the active material as well as the interband polarization describing the dipole coupling between every electron and hole lead to ultrafast carrier scattering and dephasing processes. These local interactions are then-via the dynamic coupling (i.e., induced emission and absorption processes) to the light fields - transferred to the spatial degree of freedom. During propagation the light pulse induces a dynamic spatiospectral hole burning in the charge carrier distributions. While the relaxation within the bands via carrier-carrier and carrier-phonon processes is very fast (a few hundred femtoseconds), the reestablishment of the spatial carrier distribution due to the pump current and spatial carrier transfer occurs in the nanosecond regime. The dynamics of the light fields counterpropagating in the cavity typically occurs on picosecond time scales. The large regime of relevant time scales in combination with the mutual coupling of the physical processes then leads to a complex spatiotemporal beam and gain dynamics. In a first step, the spatial hole burnt by the pulse in the spatial carrier distribution leads to an intensity-dependent carrier reduction. This spatial carrier distribution together with the intensity distribution then shapes the spatiotemporal gain available in the next time step. In particular, the laser-internal light experiences the higher carrier densities in the lateral edges. As a consequence, the diffracting light fields may laterally drift to the sides leading to a lateral broadening. Together with the reduction of the carrier density in the next time step this leads to a corresponding lateral broadening of the spatiotemporal gain. As a consequence, mediated by the laser-internal light, these changes in the spatial carrier distributions occur on a picosecond time scale.

We conclude that the presented picosecond timescale associated with the fast spatial carrier relaxation has a strong influence in particular on the emission properties of BALs. We believe that it plays an important role, e.g., for the self-mode-locking phenomena resulting in a distinct spatiotemporal picosecond emission dynamics, which is especially observed in these laterally extended devices and is associated with dynamically changing, inhomogeneous carrier distributions [17], [24].

\section{CONCLUSION AND OUTLOOK}

We have investigated the ultrafast gain dynamics of laterally extended (broad-area) semiconductor lasers. We find an ultrafast transient gain reduction and relaxation within a few picoseconds due to intraband carrier dynamics as it is common for semiconductors. The gain response after the excitation shows typical features of spectral holeburning and carrier heating. In particular, we find the slower and dominating gain relaxation due to thermal carrier relaxation lying in the range of $1.2-1.4 \mathrm{ps}$, which is slightly slower than the relaxation typically observed in semiconductor amplifiers. Local pump-probe measurements reveal in addition a lateral variation of the gain relaxation time, which can be associated to the lateral intensity and temperature distribution. Moreover, fully spatially resolved measurements show the relaxation of the combined spatiospectrally saturated gain into a spatial hole. This relaxation is connected with an effective broadening of the spatial profile of the compressed gain 
within only few picoseconds. We also find a diffusion-like spatiotemporal relaxation of the spatial hole on a timescale of several tens of picoseconds, which results from the coupling of the carrier systems to the propagating light field. Altogether, our results demonstrate that spatial and spatiotemporal effects actually occur within the ultrafast gain dynamics of these spatially extended devices. We expect that the presented first spatially resolved experiments in combination with microscopic modeling will stimulate further investigations and enhance insight into the complex spatiospectral carrier dynamics of active semiconductors, also with respect to the behavior of future ultrafast photonic devices based on spatially extended active areas.

\section{REFERENCES}

[1] K. L. Hall, G. Lenz, A. M. Darwish, and E. P. Ippen, "Subpicosecond gain and index nonlinearities in InGaAsP diode lasers," Opt. Commun., vol. 111, pp. 589-612, 1994.

[2] A. Othonos, "Probing ultrafast carrier and phonon dynamics in semiconductors," J. Appl. Phys., vol. 83, pp. 1789-1830, 1998.

[3] G. P. Agrawal and N. A. Olsson, "Self-phase modulation and spectral broadening of optical pulses in semiconductor laser amplifiers," IEEE J. Quantum Electron., vol. 25, no. 11, pp. 2297-2306, Nov. 1989.

[4] A. Uskov, J. Mørk, and J. Mark, "Theory of short-pulse gain saturation in semiconductor amplifiers," IEEE Photon. Technol. Lett., vol. 4, no. 5, pp. 443-446, May 1992.

[5] M. Y. Hong, Y. H. Chang, A. Dienes, J. P. Heritage, and P. J. Delfyett, "Subpicosecond pulse amplification in semiconductor laser amplifiers: theory and experiment," IEEE J. Quantum Electron., vol. 30, no. 4, pp. 1122-1131, Apr. 1994.

[6] R. A. Indik, R. Binder, M. Mlejnek, J. V. Moloney, S. Hughes, A. Knorr, and S. W. Koch, "Role of plasma cooling, heating, and memory effects in subpicosecond pulse propagation in semiconductor amplifiers," Phys. Rev. A, vol. 53, pp. 3614-3620, 1996.

[7] J.-Z. Zhang, J. M. Vazquez, M. Mazilu, A. Miller, and I. Galbraith, "Dispersion-induced ultrafast pulse reshaping in $1.55-\mu \mathrm{m}$ InGaAs-InGaAsP optical amplifiers," IEEE J. Quantum Electron., vol. 39, no. 11, pp. 1388-1393, Nov. 2003.

[8] E. Gehrig, O. Hess, A. Volland, G. Jennemann, I. Fischer, and W. Elsäßer, "Femtosecond dynamics of active semiconductor waveguides: microscopic analysis and experimental investigations," J. Opt. Soc. Amer. B, vol. 21, pp. 1638-1646, 2004.

[9] J. Mørk, M. L. Nielsen, and T. W. Berg, "The dynamics of semiconductor optical amplifiers, modeling and applications," Opt. Photon. News, pp. 42-48, Jul. 2003.

[10] M. S. Stix, M. P. Kesler, and E. P. Ippen, "Observations of subpicosecond dynamics in GaAlAs laser diodes," Appl. Phys. Lett., vol. 48, pp. 1722-1724, 1986.

[11] G. Eisenstein, J. M. Wiesenfeld, M. Wegener, G. Sucha, D. S. Chemla, S. Weiss, G. Raybon, and U. Koren, "Ultrafast gain dynamics in 1.5 $\mu \mathrm{m}$ multiple quantum well amplifiers," Appl. Phys. Lett., vol. 58, pp. 158-160, 1991.

[12] P. J. Delfyett, Y. Silberberg, and G. A. Alphonse, "Hot-carrier thermalization induced self-phase modulation in semiconductor traveling wave amplifiers," Appl. Phys. Lett., vol. 59, pp. 10-12, 1991.

[13] J. Mark and J. Mørk, "Subpicosecond gain dynamics in InGaAsP optical amplifiers: experiment and theory," Appl. Phys. Lett., vol. 60, pp. 2281-2283, 1992.

[14] C.-K. Sun, H. K. Choi, C. A. Wang, and J. G. Fujimoto, "Studies of carrier heating in InGaAs/AlGaAs strained-layer quantum well diode lasers using a multiple wavelength pump probe technique," Appl. Phys. Lett., vol. 62, pp. 747-749, 1993.

[15] J. A. Tatum, D. L. MacFarlaine, R. C. Bowen, G. Klimeck, and W. R. Frensley, "Ultrafast characteristics of InGaP-InGaAlP laser amplifiers," IEEE J. Quantum Electron., vol. 32, no. 4, pp. 664-669, Apr. 1996.

[16] L. Occhi, Y. Ito, H. Kawaguchi, L. Schares, J. Eckner, and G. Guekos, "Intraband gain dynamics in bulk semiconductor optical amplifiers: measurements and simulations," IEEE J. Quantum Electron., vol. 38, no. 1, pp. 54-60, Jan. 2002.

[17] T. Burkhard, M. O. Ziegler, I. Fischer, and W. Elsäßer, "Spatiotemporal dynamics of broad area semiconductor lasers and its characterization," Chaos Soliton Fract., vol. 10, pp. 845-850, 1999.
[18] P. Kürz, R. Nager, and T. Mukai, "Highly efficient phase conjugation using spatially nondegenerate four-wave mixing in a broad-area laser diode," Appl. Phys. Lett., vol. 68, pp. 1180-1182, 1996.

[19] P. P. Vasil'ev and I. H. White, "Phase-conjugation broad area twin-contact semiconductor laser," Appl. Phys. Lett., vol. 71, pp. 40-42, 1997.

[20] N. Tessler, J. Mark, G. Eisenstein, J. Mørk, U. Koren, and C. A. Burrus, "Gain dynamics in quantum well lasers and optical amplifiers: an experimental comparison," Appl. Phys. Lett., vol. 64, pp. 2050-2052, 1994.

[21] E. Gehrig and O. Hess, Spatio-Temporal Dynamics and Quantum Fluctuations in Semiconductor Lasers. Berlin, Germany: Springer-Verlag, 2003.

[22] E. Gehrig and O. Hess, "Pulse trapping and nonequilibrium spatiospectral wave mixing in broad-area semiconductor lasers," J. Opt. Soc. Amer. $B$, vol. 15, pp. 2861-2867, 1998.

[23] - "Ultrafast active phase conjugation in broad-area semiconductor laser amplifiers," J. Opt. Soc. Amer. B, vol. 18, pp. 1036-1040, 2001.

[24] J. Kaiser, I. Fischer, W. Elsäßer, E. Gehrig, and O. Hess, "Mode-locking in broad-area semiconductor lasers enhanced by picosecond-pulse injection," J. Sel. Topics Quantum Electron., vol. 10, no. 5, pp. 968-973, Sep./Oct. 2004.

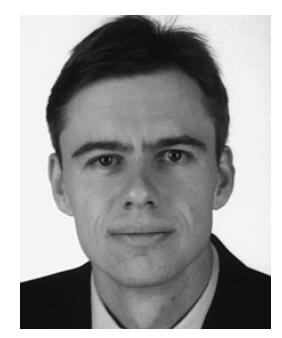

Joachim Kaiser was born in Stuttgart, Germany, in 1974. He received the diploma degree in 2000 and the Ph.D. degree in 2004 from Darmstadt University of Technology, Darmstadt, Germany.

His main research interests include spatiotemporal emission properties and femtosecond spectroscopy of broad-area semiconductor lasers and intensity noise properties of VCSELs and LEDs.

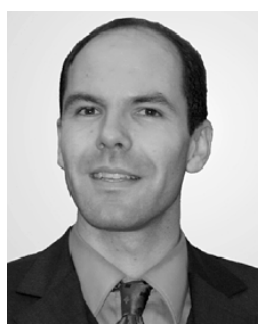

Ingo Fischer was born in Marburg, Germany, in 1966. He received the diploma and Ph.D. degrees in physics from the Philipps University, Marburg, Germany, in 1992 and 1995, respectively.

In 1995, he joined the Institute of Applied Physics, Darmstadt University of Technology, Darmstadt, Germany, where his research activities were centered around spatial, spectral, and dynamical emission properties of semiconductor lasers. His studies concentrate on nonlinear dynamics, synchronization of coupled lasers, VCSEL emission, high-power laser emission, femtosecond spectroscopy and THz generation. He has had research stays with Air Force Research Laboratories, Albuquerque, NM, in 1999, with ATR, Kyoto, Japan, in 1999 and 2000, and with UIB, Palma de Mallorca, Spain, in 2004. Since 2005, he has been with the Vrije Universiteit Brussels, Brussels, Belgium.

Dr. Fischer received the Research Prize of the Adolf-Messer Foundation in 2000 and the first Hassian Cooperation Prize of the Technology Transfer Network in 2004.

Wolfgang Elsäßer (M'94-SM'97) was born in Pforzheim, Germany, in 1954. He received the diploma degree in physics from the Technical University of Karlsruhe, Karlsruhe, Germany, in 1980, the Ph.D. degree in physics from the University Stuttgart, Stuttgart, Germany, in 1984, and the Habilitation degree in experimental physics from the Philipps-University Marburg, Marburg, Germany, in 1991.

From 1981 to 1985, he was with the Max-Planck-Institute for Solid State Research, Stuttgart, Germany. From 1985 to 1995 , he was with Philipps-University Marburg. Since 1995, he has been a Full Professor in the Institute for Applied Physics, Darmstadt University of Technology, Darmstadt, Germany. He was on sabbatical leave in 1992 with the Ecole Nationale Superieure des Telecommunications, Paris, France. His research stays have included Trinity College, Dublin, Ireland, in 1990, 1996, 1999, and 2000.

Dr. Elsäßer is a Member of the German Physical Society (DPG). He was awarded the Otto-Hahn-Medal (1985), the Werner-von-Siemens-Medal (1985), the Rudolf-Kaiser Prize (1991), and the Institute of Electrical Engineers (IEE) J. J. Thomson Premium (1995). 


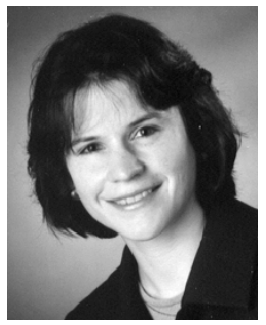

Edeltraud Gehrig obtained the diploma and Dr.rer.nat degrees in physics at the University of Kaiserslautern, Kaiserslautern, Germany, in 1995 and 1999, respectively.

She was a Senior Research Associate in the Theoretical Quantum Electronics Group, Deutsches Zentrum für Luft- und Raumfahrt (DLR) Stuttgart (2000-2003), Stuttgart, Germany. Since April 2003, she has been a Senior Research Associate at the University of Surrey, Surrey, U.K. Her research interests lie on biophotonic systems, molecular motors, ultrafast nonlinear dynamics, dynamics and control of high power semiconductor lasers and amplifiers, luminescence and quantum-dot lasers.

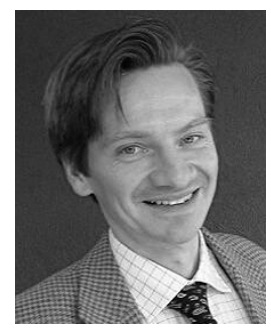

Ortwin Hess studied physics at the University of Erlangen and the Technical University of Berlin, Germany. He obtained the diploma and Dr.rer.nat degrees from the Technical University of Berlin, Berlin, Germany, in 1990 and 1993, respectively. He recevied the Habilitation degree in theoretical physics at the University of Stuttgart, Stuttgart, Germany, in 1997.

From 1990 to 1992, he was a Research Associate at Heriot-Watt University, Edinburgh, U.K., and the University of Tucson, Tuscon, AZ, and has a postdoctoral position at the University of Marburg, Marburg, Germany (1993-1994). From 1994 to 2003, he was Head of the Theoretical Quantum Electronics Group, Institute of Technical Physics of DLR, Stuttgart, Germany. He became Adjunct Professor at the University of Stuttgart in 1998. In 2001, he was an Honorary Professor at Tampere University of Technology, Tampere, Finland. He was Visiting Professor at Stanford University, Stanford, CA, (1997-1998) and the University of Munich, Munich, Germany (2000-2001). Since March 2003, he has held the Chair of Computational Quantum Electronics, Advanced Technology Institute, University of Surrey, Surrey, U.K. His research interests are focussed on the theory and mathematical modeling of the ultrafast optics and complex laser dynamics (quantum-dot lasers, control of spatiotemporal laser dynamics, ultrafast effects in active semiconductor media), nanophotonic materials (quantum dots, photonic crystals, synthetic opals, liquid crystals, biomolecules), and biomodeling, as well as nanothermodynamics and nanorheology. Together with his research group, he strives to computationally explore the terahertz-world and nanospace of novel advanced technologies in photonics as well as nano- and biomedical electronics. 